\title{
HYPOTYPOSE ET OBJECTIVATION DE LA SCÈNE DE L'HORRIBLE : LES ENJEUX DE L'ILLUSION DU RÉEL DANS QUELQUES ROMANS FRANCOPHONES CONTEMPORAINS
}

\begin{abstract}
"Hypotyposis and Objectification of the Horrible Scene: Stylizing the Figure of Reality in Some Contemporary Francophone African Novels"
\end{abstract}

SUMmaRY - This study shows that it is simplistic to argue that the narrative dynamics in contemporary Francophone African novel is monotonous. It then consists in demonstrating that it is rather indicative of a striking realism that it sometimes offers to see horrific scenes of life exhibited such that the reader immediately succumb to the temptation to share the worldview of author in review through the characters involved. Based on the operationalization of the explicit-implicit duality as structured in Pierre Barberis's sociocriticism framework, the study postulates, in two parts, that hypotyposis is a fertile and multiple variable figure which is spread at the same time like the modalisation of an event, a relevant semiological sign, a figure of the transcendence convened by the novelist in order to collect the attention of the reader by touching his psyche and causing in him a certain number of emotions such as the feeling of horror.

KEYWORDS - hypotyposis, objectification, horrible scene, sociocriticism, contemporary Francophone African novel, narration

RÉSUMÉ - La présente étude montre qu'il est réducteur de soutenir que la dynamique de narration dans le roman africain francophone contemporain est monocorde. Il s'agit alors de la monstration qu'elle est plutôt révélatrice d'un réalisme si saisissant qu'il offre parfois à voir des scènes de vie horribles exhibées telles que le lecteur succombe immédiatement à la tentation de partager la vision du monde de l'auteur en examen par le biais des personnages mis en jeu. Fondée sur l'opérationnalisation du diptyque explicite-implicite structuré à travers la sociocritique de Pierre Barbéris, l'étude postule, en deux parties, que l'hypotypose est une figure féconde à variable multiple qui se déploie à la fois comme la modalisation d'un événement, un signe sémiologique pertinent, une figure de la transcendance convoquée par le romancier en vue de capter l'attention du lecteur et de toucher sa psyché en suscitant en lui un certain nombre d'émotions, par exemple le sentiment de l'horrible. MoTs-CLÉS - hypotypose, objectivation, scène de l'horrible, sociocritique, roman africain francophone contemporain, narration

\section{Introduction}

Il serait réducteur d'avancer que le roman ne représente que des faits relatés en un temps et un lieu donnés, car " par le travail de l'écriture [il] produit un autre sens, il modifie l'équilibre antérieur du sens $»{ }^{1}$. C'est sans doute pourquoi Sartre réinvestit le code définitoire d'un écrivain dans le champ de la littérature en

\footnotetext{
${ }^{1}$ H. Mitterand, Le Discours du roman, Paris, PUF, 1980, p. 7.
} 
observant qu' « on n'est pas écrivain pour avoir choisi de dire certaines choses mais pour avoir choisi de les dire d'une certaine façon $»^{2}$. L'usage dans le récit de la figure de l'hypotypose participe de cette stratégie définissant les vrais écrivains non sans soulever la lancinante question du réalisme en création littéraire.

L'hypotypose se révèle « une description ou [...] un récit qui permettent au lecteur de se représenter un objet, un être, un paysage ou une scène, comme s'ils étaient sous ses yeux ; c'est-à-dire offrant les couleurs saisissantes de la réalité $)^{3}$. Par-delà cette définition, la présente étude commande d'autres contours épistémiques de cette figure de rhétorique tant elle s'offre à la fois comme moyen d'expression de la vraisemblance et figure propre à émouvoir. Elle suppose par ailleurs l'effacement du locuteur dans le récit. Il s'agit pour l'écrivain de « donner au lecteur l'illusion du réel grâce à des techniques $\rangle^{4}$ spécifiques.

Puisque l'hypotypose relève de ces techniques, il convient dès lors de se demander si les auteurs qui convoquent cette figure de rhétorique n'évacuent pas l'exceptionnel pour laisser voir l'illusion de la réalité observée. En d'autres termes, la figure de l'hypotypose n'est-elle pas susceptible d'agir sur l'imagination et les émotions du lecteur au regard de la vraisemblance qu'elle structure?

Le référentiel de lecture qui fonde la présente réflexion est l'épistémologie sociocritique de Barbéris. Pour ce poéticien, la sociocritique s'opère «à partir d'un effort tâtonnant et découvreur qui invente un nouveau langage, fait apparaître de nouveaux problèmes et pose de nouvelles questions $\gg{ }^{5}$. Deux stratégies de lecture ponctuent cette démarche : l'explicite et l'implicite.

\section{L'hypotypose : une stratégie narrative explicite}

Lire l'explicite renvoie aux « références claires à restituer, et qui peuvent être disséminées » ${ }^{6}$. Elle consiste à « traquer ce qui, dans le texte, se trouve dit ou dénoté $»^{7}$. Dans notre analyse, l'écriture de l'hypotypose se déploie grâce à deux facteurs explicites : la thématique de l'horreur et l'effacement du narrateur du récit.

\footnotetext{
${ }^{2}$ J.-P. Sartre, Qu'est-ce que la littérature ?, Paris, Gallimard, 1948, p. 32.

${ }^{3}$ J.-J. Robrieux, Rhétorique et argumentation, Paris, Nathan, 2000, p. 105.

${ }^{4}$ M.-F. Sculfort et al., Textes et méthodes, Paris, Nathan, 1999, p. 118.

${ }^{5} \mathrm{P}$. Barbéris, « Sociocritique », in: Introduction aux méthodes critiques pour l'analyse littéraire, Paris, Bordas, 1990, p. 124.

${ }^{6}$ P. Barbéris, « Sociocritique », op. cit., p. 137.

${ }^{7}$ Ibid.
} 


\subsection{Un espace thématique fécond}

Peintre audacieux soucieux d'exorciser par le biais de l'hypotypose la gangrène horrifiante qui ronge les sociétés africaine et américaine, l'écrivain s'appuie également sur la thématique de l'horrible pour rendre son message vraisemblable et susciter des changements de paradigmes comportementaux.

Le thème se reconnaît par sa récurrence et / ou son caractère transversal dans l'œuvre. Précisément, il « est, dans le texte, tout élément qui se répète à distance, se reconnaît semblable à lui-même, jusqu'à former une ligne explicitement significative $»^{8}$. Dans les textes que nous examinons à travers la thématique de la violence et son corollaire l'horreur, la figure de l'hypotypose dévoile plusieurs variables sémantiques lisibles dans les scènes de meurtres ainsi que d'autres faits qui illustrent, d'une part, la violation des droits de l'homme et, d'autre part, la méchanceté générique qui définit son activité rébarbative perpétrée sur son alter ego.

Tel est le cas lors des événements ressassant, dans Le Briseur de rosée d'Edwidge Danticat, l'ère des Duvalier à Haïti. Si cette période historique caractérisée par la frustration exacerbée replonge le lecteur dans les affres d'une dictature institutionnalisée par un chef d'État impitoyable, sans foi ni loi, il faut dire que les diverses stratégies traumatologiques mises en œuvre par ses hommes, dits Volontaires ou Tontons Macoutes, attestent de leur ambition affichée d'exterminer un peuple sclérosé par la récurrence des pratiques déshumanisantes. Revenant avec force détail sur l'enlèvement, l'une des tactiques punitives des élus du système dictatorial duvaliériste, la couturière Béatrice Saint Fort ouvre son cœur endolori à la curiosité de la journaliste Aline Cajuste : «ils venaient chez vous. La plupart du temps, c'était la nuit. Souvent ils venaient avant l'aube, au moment où la rosée se dépose sur les feuilles et ils vous emmenaient $»^{9}$. Les raisons pour lesquelles ils enlevaient les individus pour les torturer n'avaient pas de fondements logiques sinon subjectifs, puisque l'une d'elles pouvait simplement être la rancune. Dans le cas de Béatrice, elle fait les frais de son orgueil sentimental face aux avances infructueuses du gardien de prison. Elle précise :

Il m'a demandé d'aller danser avec lui, [...] j'ai dit non. C'est pour cela qu'il m'a arrêtée. Dans la prison, il m'a attachée à une sorte de chevalet et m'a fouettée le dessous des pieds jusqu'à ce que je saigne. Puis il m'a fait rentrer chez moi pieds nus. Sur des routes goudronnées. Sous un soleil de plomb. En plein midi ${ }^{10}$.

La haine de l'homme contre l'homme n'est pourtant pas l'apanage du roman de Danticat. Elle est également lisible dans l'imaginaire d'Eugène Ébodé qui met l'accent sur les "sanglantes équipées » qui ponctuèrent le génocide rwandais

\footnotetext{
${ }^{8}$ J.-P. Richard, Proust et le monde sensible, Paris, Seuil, 1974, p. 219.

${ }^{9}$ E. Danticat, Le Briseur de rosée, Paris, Grasset, 2004, p. 165.

${ }^{10}$ Ibid.
} 
désigné par la périphrase « moisson des têtes et des jarrets » ${ }^{11}$. Marié à une Longue nommée Dorlothée Avelimanga, le personnage de Melchior-Gaspard, issu de la tribu des Très Courts, sera froidement assassiné devant son amante alors même que les commanditaires du fratricide qui rongeait le pays lui prescrivaient d'éliminer sauvagement cette dernière, qu'il portait tant dans son cœur. Faisant de la figure de l'hypotypose le véhicule de sa communication avec son visiteur, la narratrice et rescapée Souveraine Magnifique rappelle qu'après que le couple a pendant deux jours vécu dans un état de prostration, la mort frappa à sa porte :

Leur porte ne tarda pas à voler en éclats. Le jour déclinait [...] au profit d'une petite lueur agonisante. La Longue vit entrer un homme muni de l'acier qui tuait. [...] L'assassin, déjà barbouillé de sang, regarda la Longue [...]. Elle lui tendit son cou. Il le repoussa avec dédain. Il avisa Melchior qui se tenait debout et dont la grosse tete lui faisait face. D'un geste sec et tranchant, le meurtrier fendit Melchior-Gaspard de haut en bas comme on ouvre une noix de coco $^{12}$.

\subsection{Hypotypose et énonciation : l'effacement du locuteur}

Comme le relève Robrieux, l'hypotypose « suppose l'effacement de la présence de l'énonciateur, c'est-à-dire la disparition du je, ce qui n'empêche pas la subjectivité indispensable du discours $\|^{13}$. Il s'agit alors pour nous de démontrer qu'à travers l'éclipse stratégique qui autorise le locuteur à laisser libre cours à l'objectivation d'une scène évoquée, l'attention du lecteur et le sort réservé aux personnages se trouvent prioritairement focalisés. De la sorte, la figure de l'hypotypose assume la fonction émotive. Dans la perspective de faire payer à Dorlothée son choix jugé humiliant de vivre avec un Très Court au détriment d'un Court, l'un des bourreaux saisit l'opportunité que lui offre la saison des machettes pour se venger d'elle. Le fragment qui suit révèle la violence et l'horreur du génocide suite au meurtre d'un Très Court par un Court au cœur d'un Rwanda narrativisé en proie à un indicible fratricide :

Dorlothée [...] se jeta sur les deux morceaux de son mari pour recoller l'homme achevé. Elle serra de toutes ses forces de ses mains [...] les deux bouts disjoints du pygmée qui se vidait de sang et qui était déjà mort. Après avoir traversé le corps du malheureux, la machette avait entaillé le sol. La jeune veuve resta un moment enlacée au cadavre [...] ; quand la pauvre femme comprit qu'il n'y avait plus rien d'autres à faire, elle abandonna les deux tranches du cadavre de Melchior-Gaspard ${ }^{14}$.

Dans un autre roman, Plus sage que le Roi de Béatrice Laure Mebou, nourrissant dans un royaume très lointain une haine viscérale envers tout nouveau-né de sexe masculin susceptible de lui succéder, le Roi entreprend sans succès une

\footnotetext{
${ }^{11}$ E. Ébodé, Souveraine Magnifique, Paris, Gallimard, 2014, p. 136.

${ }^{12}$ Ibid., p. 137.

${ }^{13}$ J.-J. Robrieux, Rhétorique et argumentation, Paris, Nathan, 2000, p. 105.

${ }^{14}$ E. Ébodé, op. cit., p. 58.
} 
cabale contre le très rusé garçon puisque « les petits garçons n'avaient pas le droit de vivre ${ }^{15}$. En convoquant la figure de l'hypotypose, l'écrivaine donne à voir au lecteur l'immensité de la haine nourrie par le protagoniste envers l'infortuné garçon qu'il entend tuer par noyade :

Il ordonna que l'on lui trouve un cercueil. Ce qui fut fait. Il amena Plus sage que le Roi avec lui, près de la mer. Il demanda à Plus sage que le Roi d'entrer dans un cercueil. L'intrépide enfant obéit. Puis le Roi ferma le cercueil et rentra chez lui, attendant le coucher du soleil pour venir le pousser dans la mer [...] Le roi vint donc le soir pousser le cercueil dans la mer [...]. Il rassembla alors tout le peuple et déclara que tout enfant qui oserait faire comme Plus sage que le Roi subirait le même châtiment que lui ${ }^{16}$.

Dans l'un et l'autre cas, le récit d'événements horribles se déroule sans la présence d'un locuteur s'exprimant à la première personne. Le privilège de l'histoire est ainsi laissé à un narrateur omniscient qui entraîne le lecteur dans le secret des péripéties à travers l'objectivation de la scène évoquée et la focalisation sur les personnages et leur devenir.

Mais on sait, avec Mitterand, que « tout roman propose à son lecteur, d'un même mouvement, le plaisir du récit de fiction, et, tantôt de manière explicite, tantôt de manière implicite, un discours sur le monde $»^{17}$.

\section{Implicite et hypotypose dans la dynamique de l'expression des émotions}

Débusquer l'implicite d'un texte consiste à montrer qu'il « est aussi un arcane qui dit le sociohistorique par ce qui peut ne paraître qu'esthétique, spirituel ou moral $»^{18}$. Pour dire qu'il «n'est pas fait de chose en clair et qu'on n'avait pas pu ou voulu voir $\rangle^{19}$. L'hypotypose s'inscrit en rhétorique dans la catégorie des figures de pensée, notamment les figures d'énonciation et de dialectique qui, globalement, concernent « les différentes manières de présenter un message en montrant certaines intentions plus ou moins manipulatrices, comme l'invocation $»^{20}$. S'agissant précisément de la figure de dialectique qu'est l'hypotypose, il convient d'observer que cette figure suppose un ou plusieurs interlocuteurs réels ou fictifs en vue de mettre en scène des faits quotidiens visualisés dans la perspective de les donner pour vraisemblables au lecteur.

De ce point de vue, l'hypotypose apparaît comme l'asymptote du projet rhétorique qui est, non plus seulement de persuader ou de convaincre, mais, dans un

\footnotetext{
${ }^{15}$ B. L. Mebou, Plus sage que le Roi, Yaoundé, Ifrikiya, 2009, p. 7.

${ }^{16}$ Ibid., p. 15.

${ }^{17}$ H. Mitterand, Le Discours du roman, Paris, PUF, 1980, p. 5.

${ }^{18}$ P. Barbéris, op. cit., p. 140.

${ }^{19}$ Ibid.

${ }^{20}$ Ibid., p. $99-100$.
} 
sens plus large, « de communiquer des idées ou de produire des émotions ». En d'autres termes, « il n'y a de rhétorique que lorsque la finalité du discours est bien de faire 'passer un message' $\gg$. ${ }^{21}$ Quand on relit les romans examinés, on constate que non seulement ils suscitent des émotions extrêmes chez le lecteur, mais également ils postulent un modus vivendi neuf : l'être-ensemble.

\subsection{D'une figure de rhétorique à la rhétorique des émotions figurées}

Voulant soit mettre en garde la population contre toute impossibilité d'attenter impunément à sa vie, soit intimider d'éventuels contrevenants à sa logique gouvernementale fasciste, le chef de l'État du Briseur de rosée dresse, lors d'une de ses interventions à la radio, un bilan atroce des exécutions de sang-froid « de dix-neuf jeunes officiers, membres de la garde du palais, qui, selon lui, l'avaient trahi $»^{22}$. En appelant les noms des présumés coupables, puis en répondant à chaque fois « absent $»^{23}$ avant de conclure « ils ont été fusillés » ${ }^{24}$, il cherche à frustrer le peuple et à mettre en garde tout sujet subversif qui, à l'instar du pasteur de l'Église baptiste des Anges de Bel-Air, s'attaque à son pouvoir en le défiant et en multipliant tous les dimanches sur Radio lumière des prêches acerbes réprobateurs des faits et méfaits d'un gouvernement fondamentalement redouté. À bien y regarder, une telle stratégie communicationnelle serait dénuée de sens si, dans cette occurrence au style direct, l'usage ne sécrétait pas un implicite tout en suscitant chez le lecteur de vives émotions, telles que les sentiments de frayeur et d'horreur, consécutifs à l'arrestation du pasteur dans son église. D'ailleurs cette posture se trouve renforcée quand le narrateur fait observer que : « ainsi, à cette époque, chaque ordre provenant du palais national était l'occasion de mettre à l'épreuve la loyauté de chacun, d'avertir que les choses pires encore pouvaient arriver $»^{25}$. C'est effectivement ce qui arriva au pasteur frondeur qui, lui, revendiquait tout juste plus de justice et de dignité pour l'homme :

Il fut jeté à l'arrière d'un camion. Un groupe de miliciens prit place au-dessus de lui. Il rapprocha ses pieds de sa poitrine tandis qu'ils le bousculaient d'un côté à l'autre, en le martelant de coups de crosse sur tout le corps. Son visage était à présent collé contre les ondulations du plateau métallique du camion, des semelles et des talons de chaussures pleuvaient sur lui, des mégots de cigarette étaient éteints dans ses cheveux [...]. Il reçut des décharges qui semblaient provenir d'appareils électriques portatifs qu'on appliquait contre la plante de ses pieds à présent déchaussés ${ }^{26}$.

\footnotetext{
${ }^{21}$ Ibid., p. 2.

${ }^{22}$ E. Danticat, op. cit., p. 235.

${ }^{23}$ Ibid.

${ }^{24}$ Ibid.

${ }^{25}$ Ibid.

${ }^{26}$ Ibid., p. 260-261.
} 
Emprisonné sans jamais avoir été jugé dans un lieu lugubre qu'il imagine être une cellule, le pasteur se voit couvrir les yeux avec un chiffon noir et poussiéreux, comme dans un couloir de la mort, inspirant chez le lecteur un réel sentiment de pitié. Ce sentiment se trouve renforcé quand ce lecteur examine les traitements humiliants infligés au prélat à l'aune des Droits de l'homme, notamment ceux des prisonniers : « il fut déçu de se réveiller [...]. Un liquide chaud dégoulinait sur son visage et lorsqu'il ouvrit la bouche pour se désaltérer, il se rendit compte que c'était de l'urine [...]. Il était entouré d'une demi-douzaine de prisonniers qui l'avaient arrosé de leur urine $»^{27}$. De fait, la souffrance endurée en prison inspire aux prisonniers un réel sentiment d'assistance mutuelle et de solidarité. Voilà pourquoi les prisonniers et compagnons de cellule du pasteur « avaient procédé à une sorte de guérison rituelle. Ils croyaient que leur urine [sur lui] pouvait aider à cicatriser les plaies ouvertes de son visage et de son corps $»^{28}$.

Bien qu'en prison, le pasteur n'envisage pas de mettre un terme à son militantisme ni de concéder à quiconque le droit de l'étioler, surtout pas au tortionnaire qui veut le faire passer de vie à trépas. La scène au cours de laquelle il riposte aux attaques de son bourreau et inspire au lecteur une espèce de sympathie est évocatrice : «il fit courir ses doigts sur le bord déchiqueté qui se terminait par une pointe effilée. Il saisit le morceau de bois et frappa. Il voulait viser les yeux du gros homme, mais le pic atteignit la joue droite où elle s'enfonça d'un ou deux centimètres ${ }^{29}$. La réaction du tortionnaire porte elle aussi la marque de l'hypotypose puisqu'elle sécrète une narration empreinte du sentiment d'horreur et de violence : « le gros homme se passa les mains sur le visage alors que le sang lui dégoulinait dans le cou et sur le devant de sa chemise. Il sortit son pistolet, le même P38 qu'il avait brandi devant les fidèles dans l'église, et fit feu ${ }^{30}$.

De même, dans Souveraine Magnifique d'Eugène Ébodé, le génocide rwandais qui constitue la toile de fond du récit met en scène l'extermination programmée de la race des Courts par les Longs, ces derniers se sentant en sursis dans leur propre pays. Par-delà l'horreur génocidaire que remet au jour l'écrivain camerounais par le biais de scènes réalistes, c'est davantage le sentiment de pitié qui en est induit qui frappe le lecteur. Un exemple du massacre des Longs perpétré par les Courts est donné quand l'héroïne du roman éponyme ressasse les circonstances atroces de la liquidation de ses parents par Modeste, leur ami et voisin d'hier devenu assassin :

\footnotetext{
${ }^{27}$ Ibid., p. 269.

${ }^{28}$ Ibid.

${ }^{29}$ Ibid., p. 277.

${ }^{30}$ Ibid., p. 277.
} 
Le voisin se gratta la tête. [...]. Il se ravisa et brandit sa longue et rutilante machette. [...] Il trancha la tête de mon père. [...] Ce fut horrible, car un flot de sang jaillit. Il monta jusqu'au plafond, inonda mon refuge. La tête alla s'écraser au mur. Le corps sans tête resta d'abord debout, puis tomba lourdement après des convulsions sur le carrelage $\mathrm{e}^{31}$.

À travers la figure de l'hypotypose, se déploie également dans ce roman la scénographie de l'horreur dans l'épisode qui narre le meurtre de la mère de Souveraine, la narratrice. Plus que révoltée à la vue du corps sans vie de son époux « sur ce théâtre infernal $»^{32}$, la femme qui voit périr son époux se précipite désespérément vers l'intrépide meurtrier, ne s'attendant toutefois pas au pire :

Il se dégagea. Lui déchira ses vêtements et le ventre rond de femme enceinte apparut. Et... mon Dieu, Modeste ouvrit son pantalon et demanda à ma mère de saisir sa tige... [...]. Mère a crié plus fort, elle est tombée face contre terre et a rampé vers la tête de mon père. Ses mains allaient l'étreindre. L'assassin a alors lancé son pied et tapé fort sur la tête décapitée comme on frappe dans un ballon ${ }^{33}$.

Désormais en position de force face à une femme enceinte à sa merci, Modeste s'apprête aussitôt au viol. La figure de l'hypotypose s'inscrit ainsi dans une dynamique de restitution des faits observés, sans les travestir, pour les faire agir sur la sensibilité des lecteurs :

Le voisin avait maintenant son pantalon sur les chevilles et ma mère était étalée par terre, couverte du sang de mon père. L'assassin avait les yeux exorbités. [...] Il réclamait quelque chose à ma pauvre mère. Elle s'est débattue. Elle l'a griffé, elle l'a mordu. Il lui a ouvert le ventre. Des boyaux ont giclé, mon petit frère est sorti. Un coup de machette l'a coupé en deux et un autre a tranché la tête de maman $[\ldots]$. Le sanguinaire [...] est revenu vers le cadavre de mon père. Il lui a déchiré le pantalon. Il a ricané. [...] il tenait toujours sa machette à la main. Il l'a soulevée et il a sectionné l'entre-jambe de mon père ${ }^{34}$.

Toutefois, si dans les romans africain et haïtien contemporains l'écrivain met en exergue des procédés de référence, c'est parce que le texte littéraire, à la fois langage et communication, se veut un «objet linguistique ${ }^{35}$. MinyonoNkodo partage ce point de vue quand il soutient que, «si les diverses techniques [...] révèlent l'art du romancier de choisir, de combiner, d'exploiter, des schémas formels, il reste que le roman, sous son aspect littéraire, relève fondamentalement du langage. Il est art de dire, expressivité, performance de la parole ${ }^{36}$. Le roman devient dès lors une modélisation d'un espace nouveau procédant de la réinvention d'un monde alternatif sur la base des turpitudes et des cruautés humaines

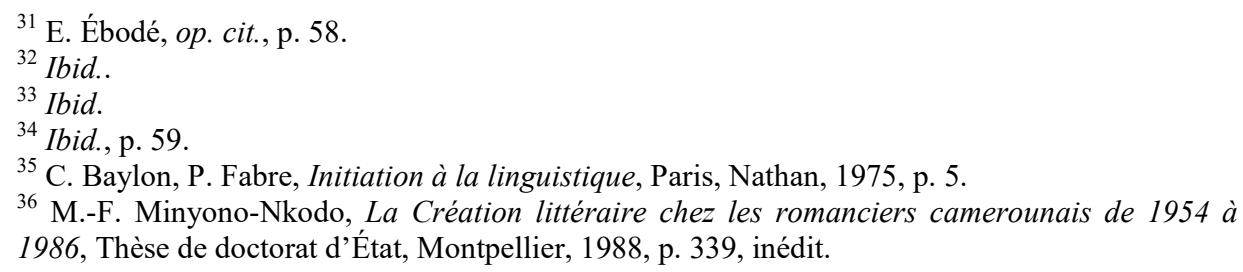


d'hier. Bien plus, il s'offre comme la postulation d'un imaginaire rénové de fond en comble, plus humaniste, d'autant qu' " écrire c'est d'abord déstructurer [...] désorganiser le monde pour tenter de le reconstruire en le représentant autrement $»^{37}$.

\subsection{De la figure de rhétorique à l'éthique de l'être-ensemble}

Par éthique, «il faut entendre l'ensemble des concessions, des convenances et des conventions qui favorisent le 'devoir-vivre-ensemble' dans la société $\rangle^{38}$. Les imaginaires africain et haïtien questionnés dans le cadre de cette modeste étude ne s'éloignent pas de la quête de cette vertu scénarisée par les écrivains. En convoquant ainsi la figure de l'hypotypose, les auteurs visent à inventer un microcosme neuf qui soit une résultante d'un double jeu entre l'écriture et les rapports sociaux violents inscrits dans la problématique de l'horreur.

Voilà pourquoi il nous semble intéressant de soutenir que, de façon implicite, les romans auscultés au moyen de l'hypotypose exhibent tous une thèse. Pour Suleiman, «le roman à thèse ou roman réaliste est fondé sur une esthétique du vraisemblable et de la représentation qui se signale au lecteur principalement comme porteur d'un enseignement, tendant à démontrer la vérité d'une doctrine politique, philosophique, scientifique ou religieuse $»^{39}$.

Ainsi, en tant qu'il est vecteur des « valeurs qui fondent l'humanisme, assurent la cohabitation, organisent la cité, et fondent le droit $»^{40}$, l'imaginaire romanesque décrypté dévoile l'idée que la violence ne saurait être la voie idéale qui mène au véritable bonheur humain. Mais elle demande à l'homme de rester vigilant s'il désire la conjurer, car « une organisation n'est jamais seulement un système de coopération, la violence et les rapports de force y sont toujours présentés et ne se réduisent pas à des pressions du système de décision $»^{41}$. Ce postulat souligne que l'être humain ne se définit pas naturellement par l'instinct belliqueux puisqu'il naît bon mais subit la perversion de la société. À ce sujet, Vergely observe : «les hommes ne sont pas moraux par nature. Mais ils ont la vocation de devenir moraux [car] naître à la vie morale, c'est naître une deuxième fois $»^{42}$.

Labellisées Gacaca ou Tribunal coutumier, les assises conviviales réunissant autour d'une même table Longs et Courts ou Très Courts à la fin du récit de Souveraine Magnifique, participent à la construction de la paix dans un Rwanda

\footnotetext{
${ }^{37}$ R. Barthes, Critique et vérité, Paris, Seuil, 1966, p. 33.

${ }^{38}$ F. Mvogo, «Littérature magrébine : quête, requête ou conquête de l'éthique ? », Annales de la Faculté des arts, lettres et sciences humaines de l'Université de Yaoundé 1, Identité culturelle et mondialisation, Yaoundé, LGE, 2008, p. 189.

${ }^{39}$ S. R. Suleiman, Le Roman à thèse ou l'autorité fictive, Paris, PUF, 1983, p. 207.

${ }^{40}$ F. Mvogo, op. cit., p. 189.

${ }^{41}$ A. Touraine, Production de la société, Paris, Seuil, 1973, p. 283.

${ }^{42}$ B. Vergely, Les Grandes interrogations morales, Toulouse, Milan, 1999, p. 26.
} 
auto-ravagé. La figure de l'hypotypose vise à exorciser la rancœur et affranchir l'homme de sa bestialité originelle. La voie du président du tribunal la convoque pour prononcer la sentence attendue d'une victime du génocide : «Modeste Constellation est reconnu coupable des faits qui lui sont reprochés. Il est condamné à sept ans de prison ferme et à cinq ans de travaux de construction d'un centre de cordonnerie $»^{43}$.

En donnant ainsi à voir par le biais de l'hypotypose le choc traumatique causé par l'homme, en scénarisant l'horrible et en suscitant chez le lecteur une vive émotion et des sentiments diversifiés tels que la pitié et / ou l'horreur, les auteurs interpellent la conscience internationale sur la menace qui pèse sur l'humanité tout entière si rien n'est fait pour exorciser la gangrène qu'est la violence. Il n'est qu'à relire la fin des romans étudiés pour se rendre compte que les écrivains, intrépides messagers de l'éthique de l'être-ensemble ${ }^{44}$, habiles et infatigables artisans de mondes toujours nouveaux, se positionnent comme des apôtres de la paix militant pour l'avènement d'un univers désinfecté, d'une solidarité humaniste agissante et d'une fraternité renégociée.

C'est ainsi qu'à la fin du récit de Plus sage que le Roi, la figure de l'hypotypose exhibe une note enchanteresse : le personnage éponyme devient un militant acharné des Droits de l'homme, mettant ainsi un terme à la barbarie sexiste d'un Roi cupide ne voulant voir naître aucun enfant mâle dans le royaume. Pour sa part, le gardien de prison tortionnaire du Briseur de rosée s'exile à New-York en monologuant : « je suis libre, [...]. Je me suis échappé ${ }^{45}$, comme pour crier victoire sur l'histoire, suite à ses méfaits en tant que rouage d'une machine de l'horreur dans laquelle il s'était engagé comme Volontaire à la solde de la dictature duvaliériste. D'ailleurs, « il n'avait plus jamais tué personne ${ }^{46}$. S'exiler signifie alors pour lui se forger une nouvelle identité et, forcément, adhérer à l'idéal de voir poindre une humanité tout aussi nouvelle. L'homme nouveau qu'il est devenu fonde une famille et sa fille Ka est symboliquement considérée comme «leur bon ange ${ }^{47}$.

Avec la dénonciation des crimes rituels dans Trop de soleil tue l'amour de Mongo Beti, la stratégie de l'hypotypose vise à subvertir le culte de l'impunité et la violation des droits de l'homme. L'intrépidité maladive de l'homme au moment de commettre un meurtre ou de causer du tort à son semblable remet au jour l'urgence pour les contemporains à penser un discours innovant en vue d'un change-

\footnotetext{
${ }^{43}$ E. Ébodé, op. cit., p. 164.

${ }^{44}$ D. Mvogo, Le Devoir de solidarité. Pour une éthique de l'être-ensemble, Yaoundé, PUCAC, 2009, p. 7. Il s'interroge ainsi sur les contours de l'être-ensemble et le déficit d'éthique dans le monde des humains : «qu'est-ce qui est fait, concrètement, pour que ici et maintenant, advienne un ordre nouveau, naisse une société nouvelle dans laquelle l'homme, pour reprendre la célèbre expression de Hobbes ne sera plus 'un loup pour l'homme'? ».

${ }^{45}$ E. Danticat, op. cit., p. 289.

${ }^{46}$ Ibid., p. 293.

${ }^{47}$ Ibid., p. 294.
} 
ment de paradigme comportemental face à la problématique de l'horreur. Voilà pourquoi le narrateur soutient sur un mode emphatique que « oui, c'est toujours calamiteux, un destin dans une république bananière, parce que le malheur n'y a jamais de fin ${ }^{48}$. Au regard du pouvoir démythifiant des mots et du rôle transformateur de la littérature dans la dynamique d'invention du monde, il y a lieu de convenir avec Minyono-Nkodo que :

La littérature africaine est fondamentalement une propédeutique ou une maïeutique à l'éthique : elle est un cadre de référence anthropologique et non un traité éthique, pour l'approfondissement du sens de la vie, de la qualité de la vie et de la vie de qualité dans le Beau, le Bien et le Bon et le Vrai ${ }^{49}$.

\section{Conclusion}

À tout prendre, l'objectivation de la scène de l'horrible par le biais de l'hypotypose revêt un double enjeu communicationnel et éthique dans les romans décryptés. À travers ses diverses variables narratives, l'hypotypose s'exhibe d'une part comme un moyen d'expression argumentatif visant à toucher la psyché du lecteur face à la problématique de l'horreur qui cause des ravages dans les sociétés qui y sont représentées. Il s'agit d'autre part d'impulser sur la base des émotions provoquées une dynamique de changement comportemental chez le lecteur, en lui présentant la réalité des faits horribles en vue de susciter son adhésion au vaste projet de voir éclore un univers neuf, moins violent. N'est-ce pas là un plaidoyer opportun lancé en vue de l'émergence d'une société éthique, moins barbare, au sein de laquelle la vertu se révélera la norme et le respect des droits humain la règle, pour le bien de tous et le bonheur de chacun?

\section{Bibliographie}

Barbéris, Pierre, "Sociocritique», in: Introduction aux méthodes critiques pour l'analyse littéraire, Paris, Bordas, p. 121-153

Barthes, Roland, Critique et vérité, Paris, Seuil, 1966

Baylon, Christian, Fabre, Paul, Initiation à la linguistique, Paris, Nathan, 1975

Beti, Mongo, Trop de soleil tue l'amour, Paris, Julliard, 1999

Danticat, Edwige, Le Briseur de rosée, Paris, Grasset, 2004

Ébodé, Eugène, Souveraine Magnifique, Paris, Gallimard, 2014

Mebou, Béatrice Laure, Plus sage que le roi, Yaoundé, Ifrikiya, 2009

Minyono-Nkodo, Mathieu-François, "L'éthique dans la littérature africaine. Contribution à la promotion d'une éducation éthique au XXI ${ }^{\mathrm{e}}$ siècle », Conférence, Mercredis des Grandes conférences, Université de Yaoundé, 5 juillet 2006, inédit

\footnotetext{
${ }^{48}$ M. Beti, Trop de soleil tue l'amour, Paris, Julliard, 1999, p. 239.

${ }^{49}$ M.-F. Minyono-Nkodo, «L'éthique dans la littérature africaine. Contribution à la promotion d'une éducation éthique au XXI ${ }^{\mathrm{e}}$ siècle », Conférence, Mercredis des Grandes conférences, Université de Yaoundé, 5 juillet 2006, p. 17, inédit.
} 
Minyono-Nkodo, Mathieu-François, La Création littéraire chez les romanciers camerounais de 1954 à 1986, Thèse de doctorat d'État, Montpellier, 1988, p. 339, inédit

Mitterand, Henri, Le Discours du roman, Paris, PUF, 1980

Mvogo, Dominique, Le Devoir de solidarité. Pour une éthique de l'être-ensemble, Yaoundé, PUCAC, 2009

Mvogo, Faustin, "Littérature magrébine : quête, requête ou conquête de l'éthique ? », Annales de la Faculté des arts, lettres et sciences humaines de l'Université de Yaoundé 1, Identité culturelle et mondialisation, Yaoundé, LGE, 2008, p. 189-197

Richard, Jean-Pierre, Proust et le monde sensible, Paris, Seuil, 1974

Robrieux, Jean-Jacques, Rhétorique et argumentation, Paris, Nathan, 2000

Sartre, Jean-Paul, Qu'est-ce que la littérature?, Paris, Gallimard, 1948

Sculfort, Marie-France, et al., Textes et méthodes, Paris, Nathan, 1999

Suleiman, Susan Robin, Le Roman à thèse ou l'autorité fictive, Paris, PUF, 1983

Touraine, Alain, Production de la société, Paris, Seuil, 1973

\section{Pierre Suzanne Eyenga Onana}

Le docteur Pierre Suzanne Eyenga Onana est enseignant-chercheur au Département de Littérature et Civilisations Africaines à la Faculté des Arts, Lettres et Sciences Humaines de l'Université de Yaoundé I, Cameroun. Ses recherches portent sur l'épistémologie de la littérature (sémiologie du texte littéraire africain et euro-américain écrit), les questions de féminismes et les Gender Studies. Il est auteur de plusieurs articles, dont «Référent temporel et volonté d'autodéfinition chez le Noiraméricain ", Annales de l'Université de Craiova, Série Sciences Philologiques, Langues et Littératures Romanes, XIX, n ${ }^{\circ} 1 / 2015$, p. 146-161 ; "Corporéité et logique des savoirs dans l'espace universitaire dans une fiction: Le Silence des déshérités de Marie Danielle Aka », Pax Academica, $\mathrm{n}^{\mathrm{o}}$ 3, 2014, p. 63-78; «Sexocide, innéité et androgynie à l'aulne du constructionnisme et de l'existentialisme beauvarien », Rhumsiki. Revue Scientifique de la Faculté des Lettres et Sciences Humaines de l'Université de Maroua, 2015. Il a participé à plusieurs séminaires et colloques : en France, au Gabon, au Cameroun, en Côte d'Ivoire. 